(2) Abderhalden: Z, physiol. Chem., 42-83 (1904-1913).

(3) U. Suzuki, Y. Matsuyama and N. Ilashimoto: Sc. Papers Inst. Physic. and Chem. Research, 4 (1926), 1.

(4) Mitchell: J. biol, Chem., 26 (1916), 231.

(5) McClendon: Proc. Soc. Exper, Biol, and Med, 28 (1930-1931), 915.

(6) W. C. Rose: J. biul. Chem., 92 (1931), ixvi.

(7) W. C. Rose: J. biol. Chem., 94 (1931), 155.

(8) Brazier: Biochem. J,, 24 (1930), 1188.

(9) Schryver and Buston: Proc. Roy. Soc. B. London, 99 (1926), 476.

\title{
Untersuchungen über die Enzyme von Bombyx mori, L.
}

III. Mitteilung. Über die Tyrosinase und

Katalase des Blutes der Seidenraupen.

$$
\text { Von }
$$

\section{Kazuo YamafuJi.}

(Aus dem Biochem. Institut der Landw. Abteilung der Kaiserl. Kyushu-Univ. au Fukwoka, Japan.)

(Eingegangen am 24, Juli 1933.)

Eingehende Angaben über die Tyrosinase und Katalase bei Insekten sind sehr selten. In Fortsetzung der Untersuchungen über die Enzyme bei den Seidenraupen, ${ }^{(1)(2)}$ teilt nun der Verfasser die Ergebnisse seine Forschungen über diese beiden Enzyme des Blutes derselben mit.

\section{1. Über die Tyrosinase.}

(A) Einricltung der Versuche:- Die für meine Versuche verwendeten Seidenraupen wurden im Frühling 1933 unter gleichen Bedingungen auf ge zogen. Die quantitative Bestimmung der Tyrosinase geschah der zahlreichen, vergleichenden Untersuchungen wegen durch die einfache, etwas modifizierte Bacusche Methode. Zu diesem $Z$ weck wurden $15 \mathrm{ccm} 0.03$ proz. 1-Tyrosinlösung, $3 \mathrm{ccm} \mathrm{M} / 3$ Phosphatpufferlösung von pH 6.64 (mit Ausnahme von Versuch 1.), $1.5 \mathrm{ccm}$ Wasser und $0.5 \mathrm{ccm}$ mit 0.85 proz. $\mathrm{NaCl}$-Lösung dreifach verdünntes Blut vermischt und 30 Minuten lang bei $30^{\circ}$ (mit Ausnahme von Versuch 2.) gehalten. Dann wurde die Reaktion durch $2 \mathrm{ccm} 10$ proż. Schwefelsäure sistiert and mit $0.01 \mathrm{~N} \mathrm{KMnO}_{4}$-Lösung bis zur Entfärbung titriert. 
(B) Optimale $p H$ :- Versuch 1. China-7-B, 2. Tag im V. Lebensalter der Raupe.

\begin{tabular}{c|c|c|c|c|c|c|c}
\hline $\mathrm{pHI}$ & 6.47 & 6.64 & 6.81 & 7.17 & 7.38 & 7.73 & 8.04 \\
\hline $\begin{array}{c}0.01 \mathrm{~N} \\
\mathrm{KMnO}_{4} \mathrm{ccm}\end{array}$ & 3.8 & 4.4 & 4.0 & 3.8 & 3.4 & 3.2 & 2.8 \\
\hline
\end{tabular}

(C) Optimaltemperatur:- Versuch 2. China-7-B, 4. Tag im V. Lebensalter der Raupe.

\begin{tabular}{c|c|c|c|c|c|c}
\hline Temp. & 23 & 30 & 37 & 45 & 55 & 65 \\
\hline $\begin{array}{c}0.01 ~ N \\
\mathrm{KMnO}_{4 .} \mathrm{ccm}\end{array}$ & 1.2 & 4.1 & 5.4 & 4.8 & 2.8 & 0.6 \\
\hline
\end{tabular}

(D) Geschlechtsuntersclied:- Versuch 3. China-7-D, 4. Tag im V. Lebensalter der Raupen.

\begin{tabular}{|c|c|c|c|c|c|c|c|c|}
\hline & \multicolumn{4}{|c|}{ 우 } & \multicolumn{4}{|c|}{$\hat{\delta}$} \\
\hline & Gruppe 1 & Gruppe 2 & Gruppe 3 & Mittel & Gruppe 1 & Gruppe 2 & Gruppe 3 & Mittel \\
\hline $\begin{array}{c}0.01 \mathrm{~N} \\
\mathrm{KMnO}_{4}, \mathrm{ccm}\end{array}$ & 2.8 & 2.8 & 3.2 & 2.9 & 4.4 & 3.8 & 4.0 & 4.1 \\
\hline
\end{tabular}

(E) Unterchied zwischen gut gewachsenen und schlecht gewachsenen Raupen :Versuch 4. Japan-110-E, 4. Tag im V. Lebensalter.

\begin{tabular}{|c|c|c|c|c|c|c|c|c|}
\hline & \multicolumn{4}{|c|}{ Gut gewachsene Raupen. } & \multicolumn{4}{|c|}{ Schlecht gewachsene Raupen. } \\
\hline & Gruppe 1 & Gruppe 2 & Gruppe 3 & Mittel & Gruppe 1 & Gruppe 2 & Gruppe 3 & Mittel \\
\hline $\begin{array}{rl}0.01 & N \\
\mathrm{KMnO}_{4} \cdot \mathrm{ccm}\end{array}$ & 3.8 & 3.8 & 4.4 & 4.0 & 3.8 & 4.4 & 3.6 & 3.9 \\
\hline
\end{tabular}

(F) Aenderungen durch Hungerzustand bei den Raupen:- Versuch 5. Europa-7-A, 4. Tag im V. Lebensalter.

\begin{tabular}{c|c|c|c|c}
\hline Hungerstunden & 1 & 6 & 24 & 50 \\
\hline $0.01 \mathrm{~N} \mathrm{KMnO}_{4} . \mathrm{ccm}$ & 1.2 & 2.4 & 1.6 & 0.8 \\
\hline
\end{tabular}

(G) Untersclied zwischen den verschiedenen Rassen der Seidenraupen :Versuch 6. V. Lebensalter. $0.01 \mathrm{~N} \mathrm{KMnO}_{4} \mathrm{ccm}$.

\begin{tabular}{|c|c|c|c|c|c|c|}
\hline Tage & Japan-110-E & Japan-110-G & China-7-B & China-7-D & Europa-7-A & Europa-7-C \\
\hline 2 & 6.4 & 6.0 & - & 3.4 & 2.8 & 0.8 \\
\hline 4 & 4.0 & 3.4 & 6.2 & 3.2 & 1.2 & 0.8 \\
\hline 7 & 4.4 & 4.0 & 10.6 & 4.8 & 2.2 & 2.4 \\
\hline
\end{tabular}


(H) Veränderungen im Laufe von drei Entwicklungsperioden von Bombyx mori, L.:- Versuch 7. Europa-7-A.

\begin{tabular}{|c|c|c|c|c|c|c|}
\hline \multirow[b]{2}{*}{ Tage } & \multirow{2}{*}{$\begin{array}{l}\text { Raupe } \\
\text { Reife }\end{array}$} & \multicolumn{2}{|c|}{ Einspinnen des Kokons } & \multicolumn{2}{|r|}{ Puppe } & $\therefore=$ \\
\hline & & 2 & 4 & 1 & 3 & 5 \\
\hline \multirow[t]{2}{*}{$\begin{array}{c}0.01 \mathrm{~N} \\
\mathrm{KMnO}_{4} \mathrm{ccm}\end{array}$} & 4.2 & 0.6 & 0.2 & 0.4 & 0.6 & 0.4 \\
\hline & \multicolumn{4}{|c|}{ Puppe } & \multicolumn{2}{|c|}{ Schmetterling } \\
\hline Tage & 8 & & & & 1 & 3 \\
\hline $\begin{array}{c}0.01 \\
\mathrm{KMnO}_{4} \mathrm{ccm}\end{array}$ & 0.3 & & & & 5.6 & 6.2 \\
\hline
\end{tabular}

\section{II. Über die Katalase.}

(A) Einrichtung der Versuche:- Als Substrat benutzte ich der gleich zeitig durchgeführten, zahlreichen Versuche und ferner der kräftigen Katalasewirkung des Bluts wegen etwas konzentrierte, nämlich ca. 0.3 Proz. $\mathrm{H}_{2} \mathrm{O}_{2}-$ Lösung. $25 \mathrm{ccm} \mathrm{H}_{2} \mathrm{O}_{2}$-Lösung, $3 \mathrm{ccm} \mathrm{M} / 3$ Phosphatpufferlösung von $\mathrm{pH} 6.64$ (mit Ausnahme von Versuch 1.), $1.5 \mathrm{ccm}$ Wasser und $0.5 \mathrm{ccm}$ mit 0.85 proz. $\mathrm{NaCl}-\mathrm{L}$ ösung dreifach verdünntes Blut wurden gemischt und nach 30 Minuten bei $30^{\circ}$ (mit Ausnahme von Versuch 2.) in Kölbchen mit 10 proz. Schwefelsäure eingelassen. Dann wurde die nicht zersetzte Menge Wasserstoffsuperoxyd duch Titration mit einer $0.01 \mathrm{~N} \mathrm{KMnO}_{4}$-Lösung bestimmt.

(B) Optimale $p H:$ - Versuch 1. China-7-B, 3. Tag im V. Lebensalter der Raupe.

\begin{tabular}{c|c|c|c|c|c|c|c}
\hline \hline $\mathrm{pH}$ & 6.47 & 6.64 & 6.81 & 7.17 & 7.38 & 7.73 & 8.04 \\
\hline $\begin{array}{c}\text { Gespaltene } \\
\mathrm{H}_{2} \mathrm{O}_{2} \mathrm{mg}\end{array}$ & 18.70 & 20.94 & 20.20 & 19.45 & 17.95 & 16.64 & 14.59 \\
\hline Spaltung \% & 24.21 & 26.81 & 26.14 & 25.18 & 23.74 & 21.30 & 18.88 \\
\hline
\end{tabular}

(C) Optimaltemperatur: - Versuch 2. China-7-B, 4. Tag im V. Lebensalter der Raupe.

\begin{tabular}{c|c|c|c|c|c|c}
\hline \hline Temp $^{\circ}$ & 5 & 15 & 23 & 30 & 37 & 45 \\
\hline $\begin{array}{c}\text { Gespaltene } \\
\mathrm{H}_{2} \mathrm{O}_{2} \mathrm{mg}\end{array}$ & 11.05 & 14.28 & 15.13 & 11.56 & 8.84 & 7.17 \\
\hline Spaltung \% & 14.30 & 18.49 & 19.59 & 14.96 & 11.44 & 9.24 \\
\hline
\end{tabular}


(D) Geschlechtsunterschied:- Versuch 3. China-7-D, 4. Tag im V. Lebensalter der Raupen.

\begin{tabular}{|c|c|c|c|c|c|c|c|c|}
\hline & \multicolumn{4}{|c|}{ q } & \multicolumn{4}{|c|}{$\hat{\delta}$} \\
\hline & $\begin{array}{c}\text { Gruppe } \\
1\end{array}$ & $\begin{array}{l}\text { Gruppe } \\
\quad 2 \\
\end{array}$ & $\begin{array}{c}\text { Gruppe } \\
3\end{array}$ & Mittel & $\begin{array}{c}\text { Gruppe } \\
1\end{array}$ & $\begin{array}{c}\text { Gruppe } \\
2\end{array}$ & $\underset{3}{\text { Gruppe }}$ & Mittel \\
\hline $\begin{array}{c}\text { Gespaltene } \\
\mathrm{H}_{2} \mathrm{O}_{2} \mathrm{mg}\end{array}$ & 14.21 & 13.09 & 13.46 & 13.59 & 17.95 & 17.58 & 18.70 & 18.08 \\
\hline Spaltung \% & 18.40 & 16.82 & 17.43 & 17.55 & 23.74 & 22.75 & 24.21 & 23.57 \\
\hline
\end{tabular}

(E) Unterschied zwischєn gut gewachsenen und schlecht gewachsenen Raupen :Versuch 4. China-7-D, 7. Tag im V. Lebensalter.

\begin{tabular}{|c|c|c|c|c|c|c|c|c|}
\hline & \multicolumn{4}{|c|}{ Gut gewachsene Raupen } & \multicolumn{4}{|c|}{ Schlecht gewachsene Raupen } \\
\hline & $\begin{array}{c}\text { Gruppe } \\
1\end{array}$ & $\begin{array}{c}\text { Gruppe } \\
2\end{array}$ & $\begin{array}{c}\text { Gruppe } \\
3\end{array}$ & Mittel & $\begin{array}{c}\text { Gruppe } \\
1\end{array}$ & $\begin{array}{c}\text { Gruppe } \\
2\end{array}$ & $\begin{array}{c}\text { Gruppe } \\
3\end{array}$ & Mittel \\
\hline $\begin{array}{l}\text { Gespaltene } \\
\mathrm{H}_{2} \mathrm{O}_{2} \mathrm{mg}\end{array}$ & 16.08 & 15.71 & 15.33 & 15.77 & 13.46 & 12.72 & 12.34 & 12.84 \\
\hline Spaltung \% & 20.82 & 20.33 & 19.75 & 20.30 & 17.43 & 16.46 & 15.97 & 16.62 \\
\hline
\end{tabular}

(F) Änderungen durch Hungerrustand bei den Raupen:- Versuch 5. Europa-7-C, 4, Tag im V. Lebensalter.

\begin{tabular}{c|c|c|c|r}
\hline \hline Hungerstunden & 1 & 6 & 24 & \multicolumn{1}{|c|}{50} \\
\hline Gespaltene $\mathrm{H}_{2} \mathrm{O}_{2} \mathrm{mg}$ & 7.48 & 7.85 & 6.73 & 8.23 \\
Spaltung $\%$ & 9.68 & 10.17 & 8.71 & 10.65 \\
\hline
\end{tabular}

(G) Unterschied zwischen den verschiedenen Rassen der Seidenraupen:Versuch 6. V. Lebensalter.

\begin{tabular}{c|r|r|r|r|r|r|r}
\hline Tage & \multicolumn{2}{|c|}{2} & \multicolumn{2}{|c|}{4} & \multicolumn{2}{|c}{7} \\
\hline Rasse & $\begin{array}{r}\text { Gespaltene } \\
\mathrm{H}_{2} \mathrm{O}_{2} \mathrm{mg}\end{array}$ & $\begin{array}{c}\text { Spaltung } \\
\%\end{array}$ & $\begin{array}{l}\text { Gespaltene } \\
\mathrm{H}_{2} \mathrm{O}_{2} \mathrm{mg}\end{array}$ & $\begin{array}{c}\text { Spaltung } \\
\%\end{array}$ & $\begin{array}{c}\text { Gespaltene } \\
\mathrm{H}_{2} \mathrm{O}_{2} \mathrm{mg}\end{array}$ & $\begin{array}{c}\text { Spaltung } \\
\%\end{array}$ \\
\hline Japan-110-E & 16.08 & 20.82 & 14.96 & 19.30 & 15.71 & 20.33 \\
Japan-110-G & 24.31 & 31.47 & 20.19 & 26.14 & 19.82 & 25.66 \\
China-7-B & & & 10.71 & 13.86 & 15.47 & 20.03 \\
China-7-D & 19.89 & 25.75 & 13.47 & 17.44 & 14.59 & 18.89 \\
Europa-7-A & 8.16 & 10.56 & 6.68 & 8.65 & 7.85 & 10.16 \\
Europa-7-C & 6.29 & 8.14 & 7.48 & 9.68 & 10.10 & 13.07 \\
\hline
\end{tabular}

(H) Veränderungen im Laufe con drei Entwicklungsperioden von Bombyx mori, L.:- Versuch 7. Europa-7-A. 


\begin{tabular}{|c|c|c|c|c|c|c|}
\hline & \multirow{2}{*}{$\frac{\text { Raupe }}{\text { Reife }}$} & \multicolumn{2}{|c|}{ Einspinnen des Kokons } & \multicolumn{3}{|c|}{ Puppe } \\
\hline Tage & & 2 & 4 & 1 & 3 & 5 \\
\hline $\begin{array}{l}\text { Gespaltene } \\
\quad \mathrm{H}_{2} \mathrm{O}_{2} \mathrm{ng}\end{array}$ & 11.22 & 21.32 & 20.94 & 19.82 & 40.02 & 31.56 \\
\hline \multirow[t]{2}{*}{ Spaltung $\%$} & 14.52 & 27.60 & 27.09 & 25.66 & 51.81 & 40.85 \\
\hline & \multicolumn{4}{|c|}{ Puppe } & \multicolumn{2}{|c|}{ Schmetferling } \\
\hline Tage & 8 & \multicolumn{2}{|c|}{11} & 13 & 1 & 3 \\
\hline $\begin{array}{l}\text { Gespaltene } \\
\quad \mathrm{H}_{2} \mathrm{O}_{2} \mathrm{mg}\end{array}$ & 35.36 & \multicolumn{2}{|c|}{14.11} & 11.97 & 18.50 & 25.57 \\
\hline Spaltung $\% \%$ & 45.77 & \multicolumn{2}{|c|}{18.27} & 15.50 & 23.95 & 33.10 \\
\hline
\end{tabular}

\section{Zusammenfassung.}

(1) Die Tyrosinase des Bluts von Bombyx mori wirkt am besten bei pH 6.6. Die Optimaltemperatur liegt bei $37^{\circ}$.

(2) Die Tyrosinasewirkung des Bluts ist bei den Männchen etwas stärker als bei den Weibchen, aber es besteht kein Unterschied zwischen den gut gewachsenen und den schlecht gewachsenen Raupen. Durch Hunger wird die Wirkung der Tyrosinase erst etwas stärker und dann allmählich schwächer.

(3) Der Tyrosinasegehalt des Bluts der japanischen und der chinesischen Rassen ist recht viel höher als derjenige der europäischen. Bei den reifen Larven ist die Tyrosinase sehr aktiv, aber mit dem Beginn des Spinnens der Kokons tritt eine rapide Abnahme auf und während der Puppenperioden bleibt sie bedeutend zurück. Dann wieder vermehrt sich die Tyrosinase in den Tagen des Schmetterlingslebens rasch bis zu einem Maximum.

(4) Die Blutkatalase von Bombyx mori zeigt die optimale Aktivität bei $\mathrm{pH} 6.6$; ihr Temperaturoptimum liegt bei $23^{\circ}$.

(5) Die Katalasewirkung des Bluts ist bei den Männchen etwas stärker als bei den Weibchen und auch bei gut gewachsenen Raupen etwas höher als bei schlecht gewachsenen. Es wurde aber innerhalb der zwei Hungertage keine Änderung beobachtet.

(6) Der Katalasegehalt-des Bluts der japanischen und der chinesischen Rassen ist recht viel höher als derjenige der europäischen. Beim Beginn des Kokonspinnens der reifen Larve findet eine rapide Zunahme der Katalaseaktivität statt, die ihr Maximum meist am dritten Tage der Puppenperiode erreicht. Dann sinkt die Katalase allmählich und wird in der Periode des Schmetterlingslebens wieder bedeutend stärker. 


\title{
Literatur.
}

(1), ( 2 ) K. Yamafuji: I u. II. Mitteilung, Bull. Agr. Chem. Soc. Japan, 8, 156, (1932); 9, 19, (1933).

\section{On the Two Kinds of Saponin of Soya Bean.}

By

\author{
Koji Orano and Iwao OHara.
}

(Received Oct. 21, 1933)

We have isolated easily and in large quantity the two kinds of saponin, crystal and amorphous, from the alcoholic extraction of soya bean as the byproduct in the process of the alcoholic extraction method of the soya bean oil. (1) The crystalline saponin had been investigated by Y. Sumiki ${ }^{2)}$ already and he reported that it formed squama, melting at $222 \sim 4^{\circ}$, the molecular formula was $\mathrm{C}_{49 \sim 52} \mathrm{H}_{70 \sim 54} \mathrm{O}_{21}$, by the hydrolysis sapogenin $\left(\mathrm{C}_{32 \sim 35} \mathrm{H}_{4 \xi \sim 58} \mathrm{O}_{3}\right)$, glucose, rhamnose, arabinose and other unknow acidic substance were obtained, and its haemolytic and toxic power were very weak. E. Walz $z^{3}$ had reported that there were three kinds of saponin in soya bean, but those details remained unexplained. We have compared the two saponins and further investigated them closely as the first step toward the utilization of alcoholic extract.

\section{Experimental.}

1) Isolation:- Alcoholic extract of soya bean, from which the bean oil was separated and alcohol was evaporated, is mixed with $\mathrm{NaCl}$-solution and from separated precipitate the ether soluble matter is removed, then the residue is recrystallized from $80 \%$ alcohol repeatedly and crystal and amorphous ones are separated by fractional crystallization.

2) Molecular formulas and properties:-

(A) Crystalline saponin. mel. pt. $225 \sim 227^{\circ}$. Its properties are identical with those of Sumiki and natrium-salt is hexagonal plate, decomposing at $259^{\circ}$, both have not haemolytic power.

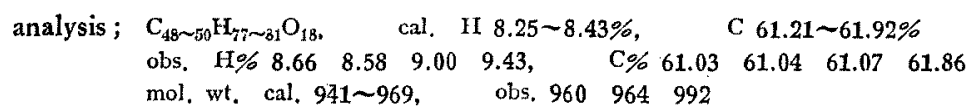

(B) Amorphous saponin. mel. pt. $216 \sim 218^{\circ}$. White powder is recry- 